\title{
Proceedings
}

\section{Analytical Model of Double Barrier THz Rectifier ${ }^{\dagger}$}

\author{
Fabrizio Palma * and Rosario Rao \\ Rome University La Sapienza, Rome, Italy; rosario.rao@uniroma1.it \\ * Correspondence: fabrizio.palma@uniroma1.it; Tel.: +34-934-039-876 \\ † Presented at the Eurosensors 2017 Conference, Paris, France, 3-6 September 2017.
}

Published: 31 August 2017

\begin{abstract}
The application of the new structure to the complex field of $\mathrm{THz}$ receivers requires the availability of an analytical model, reliable and able to highlight the dependence on the parameters of the physical structure. We present a model of the rectification capability of the double barrier Terahertz radiation $(\mathrm{THz})$ detector, a CMOS compatible structurer. The model is based on the hydrodynamic semiconductor equations; solved in the small signal approximation. The model highlights the high frequency dynamics of carriers in the structure; and furnishes new insight on the origin of the self-mixing rectification process.
\end{abstract}

Keywords: Terahertz; rectification; self-mixing; hydrodynamic

\section{Introduction}

Terahertz radiation $(\mathrm{THz})$ cover the gap between the microwaves and the infrared regions. Some peculiar characteristics of the $\mathrm{THz}$, like strong penetrability, sensitivity to chemical bonds, higher resolution, make $\mathrm{THz}$ an attractive instrument in a great number of applications, as medical imaging, and security check. A strong effort was carried on in order to achieve $\mathrm{THz}$ sensors by using the standard, low cost, CMOS technology. CMOS technology offer reproducibility and would permit to achieve arrays of detector, that once used in panels could even permit a large area approach.

We recently proposed a new structure of $\mathrm{THz}$ detector [1], based on the CMOS image sensor technology obtained by the combination of a $\mathrm{n} / \mathrm{p}+$ barrier and a $\mathrm{p}+/$ metal Schottky barrier (in the following referred as double barrier), Figure 1 . The asymmetry between the two barriers determines the rectifying capability. The reported TCAD simulations of the double barrier structure indicated that the detection capability is preserved up to very high frequency range [1].

Analytical models of $\mathrm{THz}$ rectification, available in literature, are mainly based on lamped equivalent circuits, and show in general $1 / \omega^{2}$ dependence above a very low cutoff frequency [2].

In contrast with these approaches, Monte Carlo simulations [3] demonstrated how in Schottky diodes, the Capacitance/Voltage $(\mathrm{C}-\mathrm{V})$ nonlinearity dominate with respect to the Current/Voltage (I-V) nonlinearity in the range $0.2-5 \mathrm{THz}$.

A models which may drive the design and the applications of the new structure is highly desirable. In this paper we present an analytical model of the carrier's dynamic into the double barrier detector, based on the hydrodynamic semiconductor equations, solved in the small signal approximation. The self-mixing process, which give rise to the $\mathrm{THz}$ rectification, is related to the nonlinear effect of the carrier dynamic within the depletion layer. In our approach, we are able to highlight different contributions coming from different portions of the two barriers structure. The carrier's dynamic also furnishes the frequency behavior of the rectification. 


\section{Analytical Model}

A sketch of the double barrier structure is reported in Figure 1. At the left border of the depletion region of the n-type layer is placed an ohmic (cathode). A $n / p+$ junction occurs at $X=0$ and $\mathrm{x}=\mathrm{W}_{\mathrm{n}}$ are the extremes of the depletion region of the n-type silicon, assumed in the sharp step depletion approximation. The $\mathrm{p}+$ region has a thickness $\mathrm{D}$, and it is assumed as completely depleted. The $\mathrm{p}+$ region and the metal result in a Schottky barrier at $\mathrm{x}=\mathrm{W}=\mathrm{w}_{\mathrm{n}}+\mathrm{D}$, forming the anode contact.

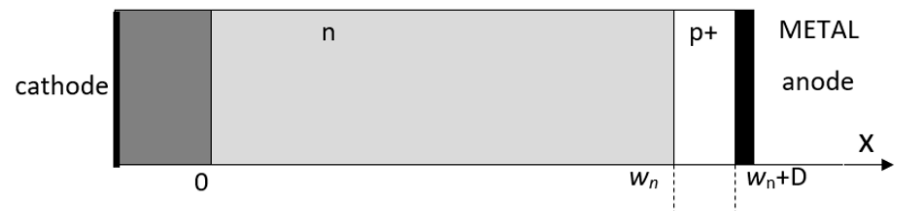

Figure 1. Schematic description of double barrier structure.

Charge balance, and the built-in potential across the structure due to the difference between the Fermi level in the $\mathrm{n}$ layer and the metal Work function, permit a straightforward calculation of the potential distribution across the structure. The detector is assumed in the zero bias condition, as expected in a detector, in order to reduce the junction noise.

Assuming the zero bias condition, and the zero potential at $x=0$, the electron density is given by:

$$
\mathrm{n}_{0}(\mathrm{x})=\mathrm{n}_{\mathrm{n} 0} \mathrm{e}^{-\frac{\phi(\mathrm{x})}{\mathrm{k}_{\mathrm{B}} \mathrm{T}}}
$$

where $\phi(\mathrm{x})$ is the potential drop, $\mathrm{k}_{\mathrm{B}}$ the Boltzman constant, and $\mathrm{T}$ the absolute temperature. Figure 2 reports the behavior of $n_{0}$ inside the structure.

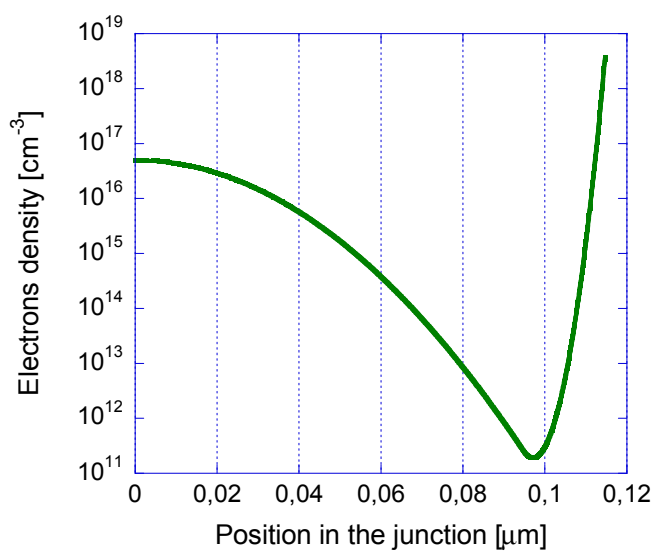

Figure 2. Electron density as a function of position in the structure.

When a sinusoidal radiofrequency voltage drop, $V_{R F}(t)=\hat{v}_{R F} e^{-j \varpi t}$ is applied to the structure, a sinusoidal variation of all the physical quantities can be assumed. The hydrodynamic semiconductor equations, must be solved, in the small signal approximation, within the depletion layer of the junction. The delay due to the kinetic of electron, accelerated by the electric field, is described by the Euler equation limited by the effect of electron collision where $\tau$ is the collision time [4,5].

We assume a spatially constant electric field along the depletion layer, as a consequence also the amplitude of the first order electron velocity variation must be assumed constant.

Assuming the first order variations of the electron density has a sinusoidal behavior in time: $\mathrm{n}(\mathrm{x}, \mathrm{t})=\hat{\mathrm{n}}(\mathrm{x}) \mathrm{e}^{-\mathrm{j} \omega \mathrm{t}}$, and variations of the electron drift velocity constant in space and sinusoidal in time: $v(t)=\hat{v} e^{-j \omega t}$, the semiconductor equations for the variations can be expressed as following:

$$
\widehat{\mathrm{E}} \cong \hat{\mathrm{v}}_{\mathrm{RF}} / \mathrm{W}
$$




$$
\begin{gathered}
\hat{v}=\frac{\frac{e}{m} \widehat{E}}{j \omega+\frac{1}{\tau}} \\
j \varpi \hat{n}=-\hat{v} \frac{\partial n_{0}}{\partial x}+D_{n} \frac{\partial^{2} \hat{n}}{\partial^{2} x}
\end{gathered}
$$

where Equation (2) derives from the Poisson equation, Equation (3) derives from the Euler equation, and Equation (4) derives from the electron continuity equation taking into account both the contributions due to transport and diffusion. $\mathrm{e}$ is the electron charge, $\mathrm{m}$ the electron mass, $\tau$ the electron collision mean time, $D_{n}$ the electrons diffusion coefficient. Two asymptotic expressions can be calculated as reported by:

$$
\begin{aligned}
& \widehat{\mathrm{n}}_{\mathrm{A}}=-\frac{\widehat{\mathrm{v}} \frac{\partial \mathrm{n}_{0}}{\partial \mathrm{x}}}{\mathrm{j} \omega} \text { neglecting the term: } \frac{\partial^{2} \widehat{\mathrm{n}}}{\partial^{2} \mathrm{x}}
\end{aligned}
$$

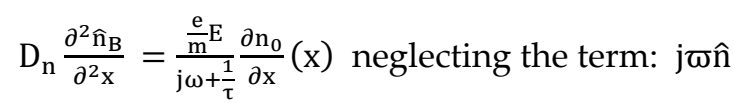

$\hat{\mathrm{n}}_{\mathrm{A}}$ has the same spatial distribution of $\frac{\partial \mathrm{n}_{0}}{\partial \mathrm{x}}$ and a strong frequency dependence. Below the collision frequency, $\hat{\mathrm{n}}_{\mathrm{B}}$ solution is not depending on the frequency.

The choice between the two solutions $\hat{\mathrm{n}}_{\mathrm{A}}$ and $\hat{\mathrm{n}}_{\mathrm{B}}$, can be done choosing the largest contribute to the Equation (4) according to different operators.

Electrons density variations contribute to the nonlinear second term of the transport equation: $\mathrm{J}_{\mathrm{n}}=$ env, giving rise to a DC self-mixing term, $\mathrm{J}_{\mathrm{DC}}=\langle\mathrm{env}\rangle$, i.e., the time average of the product. We note that only the product between first order terms in phase gives a DC term not null. In particular, it results that the contribution of solution $\hat{n}_{A}, J_{D C} A=\left\langle n_{A} v\right\rangle$, is null. On the contrary solution $\hat{\mathrm{n}}_{\mathrm{B}}$, is practically in phase with applied RF voltage, and represents the main contribution to the DC self-mixing.

The current due to the self-mixing process on its own gives rise to a potential drop contributes across the structure. Total potential can be obtained by the integration of these contributions.

\section{Model Results and Comparison with Numerical Simulations}

The AC model depicts the mechanisms of the THz modulation of the charge in the depleted regions of the structure. Comparison with TCAD simulations permits to validate the model assumptions. In Figure 3a, the distributions of real (red lines) and imaginary (green lines) parts of variations of electron density are reported, as obtained from the combination of the two asymptotic solutions $\hat{\mathrm{n}}_{\mathrm{A}}$ and $\hat{\mathrm{n}}_{\mathrm{B}}$, and are compared in Figure $3 \mathrm{~b}$ with the same quantities obtained with the TCAD simulator, at the frequency of $1 \mathrm{THz}$. Both the approaches indicate that the ratios between the real and the imaginary parts exchange within the depletion layer, corresponding in the model at the exchange between the two asymptotic solutions. The discontinuity in the model is justified by asymptotic approach of the solution. In this simulation the $\mathrm{n}$ is doped $5 \times 10^{16} \mathrm{~cm}^{-3}$.

Figure 4 reports the distribution of the potential drop inside the depletion region obtained by the proposed model. Self-mixing only occurs in a portion of the depleted $\mathrm{n}$ region. The contribution of the region $\mathrm{n}$ is normally much larger than the contribution of the thin $\mathrm{p}+$ doped region.

Figure 5 reports the frequency dependence of the rectification voltage across the structure, for three doping concentrations. The lower doping case shows an inversion of the potential polarity due to the abrupt decrease of the self-mixing process in the $n$ doped region, with upper hand of the contribute of the $\mathrm{p}+$ doped region. 


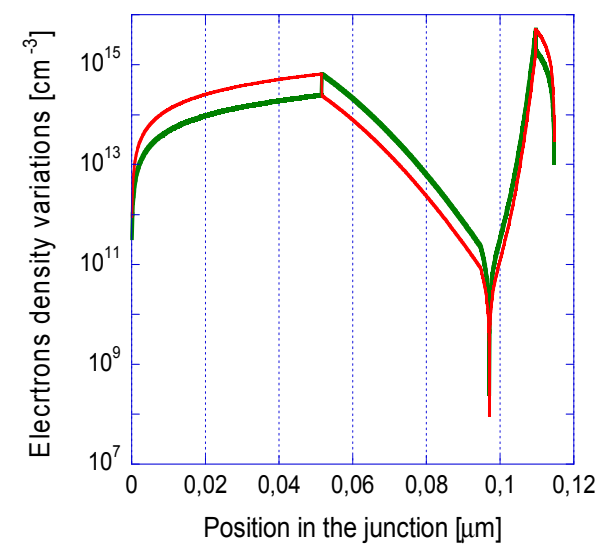

(a)

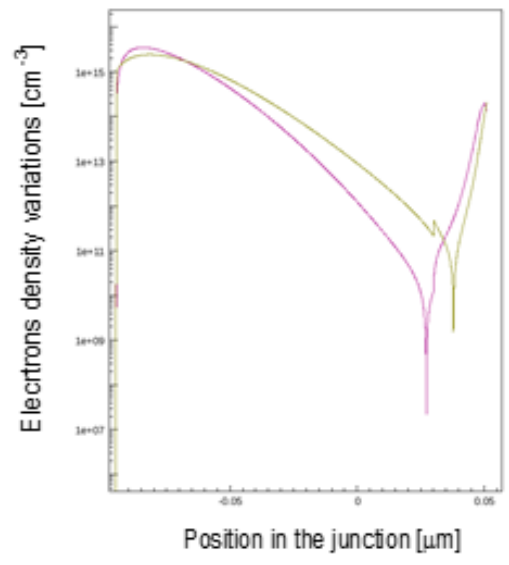

(b)

Figure 3. Distribution of real (red lines) and imaginary (green lines) parts of variations of electron density at $1 \mathrm{THz},(\mathbf{a})$ as obtained from the two solutions $\hat{\mathrm{n}}_{\mathrm{A}}$ and $\hat{\mathrm{n}}_{\mathrm{B}}$, and (b) from TCAD simulations.



Figure 4. The distribution of the self-mixing potential within the junction.

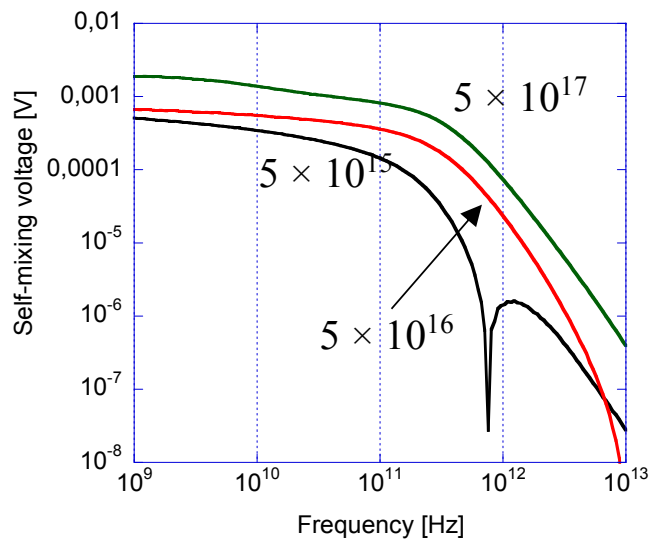

Figure 5. The frequency behavior of the DC self-mixing voltage generated through the structure, with different values of doping of the $\mathrm{n}$ layer in $\mathrm{cm}^{-3}$.

\section{Conclusions}

We present an analytical model of the carrier's dynamic into the double barrier detector, based on the hydrodynamic semiconductor equations, solved in the small signal approximation. Parameter and frequency dependence were illustrated. 
Conflicts of Interest: The authors declare no conflict of interest.

\section{References}

1. Varlamava, V.; De Amicis, G.; Del Monte, A.; Perticaroli, S.; Rao, R.; Palma, F. CMOS-Compatible Room-Temperature Rectifier Toward Terahertz Radiation Detection. J. Infrared Milli Terahz Waves 2016, 37, 737. doi:10.1007/s10762-016-0265-x.

2. Varlamava, V.; Palma, F.; Nenzi, P.; Balucani, M. Electric Field Enhancement in 3-D Tapered Helix Antenna for Terahertz Applications. IEEE Trans. Terahertz Sci. Technol. 2014, 4, 360-367. doi:10.1109/TTHZ.2014.2310122.

3. Shashkin, V.I.; Vostokov, N.V. Sensing microwave-terahertz detectors based on metal-semiconductor-metal structures with symmetrical I-V characteristic. IEEE J. Electron Devices Soc. 2013, 1, 76-82. doi:10.1109/JEDS.2013.2252235.

4. Shiktorov, P.; Starikov, E.; Gruzinskis, V.; Pérez, S.; González, T.; Reggiani, L.; Vaissiere, J.C. Monte Carlo simulation of Schottky diodes operating under terahertz cyclostationary conditions. IEEE Electron Device Lett. 2004, 25, 1-3. doi:10.1109/LED.2003.821635.

5. Dyakonov, M.; Shur, M. Detection, mixing, and frequency multiplication of terahertz radiation by two-dimensional electronic fluid. IEEE Trans. Electron Devices 1996, 43, 380-387. doi:10.1109/16.485650.

(C) 2017 by the authors. Licensee MDPI, Basel, Switzerland. This article is an open access article distributed under the terms and conditions of the Creative Commons Attribution (CC BY) license (http://creativecommons.org/licenses/by/4.0/). 